\title{
Exponential Function in Physics Education from the view of Knowledge in Pieces Theory
}

\section{Peter Demkanina $^{a}$ Karolína Šromekováb ${ }^{\text {, Adam Slovák }}$}

Comenius University in Bratislava, Faculty of Mathematics, Physics and Informatics, Department of Education, Slovakia, corresponding author: peter.demkanin@uniba.sk

\begin{abstract}
Learning is deeply rooted in the social environment and can flourish in a well-designed learning environment. Participative teaching-learning requires activities set in many different contexts, developing the grain-size pieces of knowledge and chaining fragments by causal chaining, time sequences and mathematical similarities. Based on the Knowledge-in-Pieces theory by diSessa, we focus on the development of the ability to think in terms of the exponential function, in Physics education from the age of 12. Main ideas are supported by experience with one activity. The activity is focused on topics such as measuring quantity, unit, method of measurement. Pupils propose which features of the ball can be investigated and measured. After this introduction, a quantity related to the bouncing is put forward. It is a dimensionless quantity, which pupils can express as a percentage. Pupils empirically investigate bouncing of a ball. In the extension, at a higher age, the graph of maximal height vs the number of bounces, pupils discover the same shape they have seen in a water-cooling experiment. The activity was tested on a small sample of pupils, and some interesting results have been noticed. At the end of this contribution, we offer a list of other contexts naturally involving the exponential shape graph applicable in science education.
\end{abstract}

Keywords: physics education, knowledge in pieces, exponential function, bouncing ball

\section{Introduction}

In this article we are presenting our vision of an example of a teaching-learning sequence based on The Learning Science movement presented, e.g. by diSessa (2014) and Sawyer 
(2014). Some aspects of the framework have been published in a book (Demkanin 2018). In the background, we bring some points we have not presented in previous papers, and mostly we refer to Sfard and Cobb (Sfard \& Cobb, 2014). Here we focus on one example, the exponential function. We apply the theoretical background to one teaching-learning sequence devoted to the introduction of physical quantities and units of physical quantities at the age of 12, formal education, physics (science). The sequence is set to the context of a bouncing ball. We also bring some experience with the pilot use of this activity.

\section{Background}

This article is rooted in the theoretical background of Knowledge-in-Pieces theory.(diSessa, 2014) developed on the idea that naïve physics is composed of a thousand elements. For these elements, diSessa uses the term phenomenological primitives (p-prims). P-prims in the mind of a person (e.g. a pupil) cause, which phenomena the person senses as natural and which surprising. P-prims are only weakly organised and often are significantly dependent on the context. Within the Knowledge-in-Pieces theory, the elements (grain size elements) are chained by causal chaining, time sequencing and by mathematical similarities. P-prims are linked together at a higher level - a coordination class, and coordination classes together with mental models and other structures form the cognitive ecology of the mind of a person.

Knowledge-in-Pieces (KiP) theory offers principles for a teaching-learning sequence design (including design of the lessons and whole course). We can apply these principles to both, re-construction of knowledge and development of new knowledge. Re-construction of knowledge we use in topics where the pupil has a naïve intuitive working theory (e.g. forces acting on an object immersed in water - Archimedes principle). Development of a new knowledge we apply in topics where the pupil has no pre-concepts, no experience (e.g. movement of an electron in an electromagnetic field - Thomson cathode-ray experiment). Within the re-construction, KiP principles offer a new, well-planned experience to add a missing knowledge (not the suppression of previous). Development of concepts in a topic where the pupil has no experience is based on acquiring grain-size pieces of knowledge.

Within the Knowledge-in-Pieces theory, teachers are lead to look at the concept formation during the concept formation itself, within short time sequences. The bits of the curriculum are so small that the teacher can sense and analyse the process of learning just during learning. The teacher can offer formative feedback quite precisely and instantly. We are not analysing the pupils only before and after the teaching-learning sequence, but also during 
the sequence. The teacher sees the differences among the pupils and has means to adjust teaching for individuals.

To build a knowledge related to a phenomenon, to develop skills related to scientific thinking and working, and to develop a working theory at the level of the pupil, the pupil must have experience within various contexts. The contexts are introduced by both pupils and the teacher. Pupils initiate learning within the contexts directly related to their everyday experience and the teacher initiates the relevance of those contexts, which are a little bit further from the everyday experience. Learning is making sense of new experience by learners in collaboration with others (Harlen, 2006). Experiences must be organised into a functional system. It requires some time. This is one of the ideas developed later in this article, on the topic of exponential dependence.

At this time, in Slovakia, and also in other educational systems, the most widespread is the acquisition system, based on Piagetian constructivism. In this system, the teacher is a provider of information, provider of the opportunities to learn. The role of a teacher is weakened, pupils gain knowledge via their own learning, via personal activity. A kernel of this approach of teaching is the belief, that physics is a system of existing structures, and the role of the pupil is to get these structures. In contrast to the acquisition system, a participative approach is emerging in more countries. In the participative approach, a pupil is set to the time and culture, where he or she lives. Physics, science and mathematics are considered as forms of human activity. Within the participative approach, the existing structures and existing information are sources for pupil's activity. One of the roots of this approach is the theory of Vygotsky, in which the learning has been looked at as gaining abilities to perform human activities. In Slovakia, the well-known approach to mathematics education has been developed by Hejny (2012).

Pupils live in a culture. A pupil lives within an environment, which includes sources of information, experts, tools (including software), and available services. It is up to the teacher and the school which of the environmental components incorporate into the teaching-learning environment. The teaching-learning environment usually contains also outdoor places and various social contexts.

\section{Activity - "How to measure a ball?" - intended curriculum}

As we have presented in the introduction, we present a design of an example of a teachinglearning sequence (one lesson activity) for formal education, physics (science), for the age of 12. The activity is intended at the beginning of physics education, which is in Slovakia at the age of 12. Just one lesson before this activity, pupils measure the temperature of various 
objects in the school laboratory, including pupil's hands and graph measured temperature pupils move the temperature sensor from hot water to cold water and back and look at the changes in the measured temperature.

\subsection{Big Idea of the activity}

Within this activity, we intended to develop the same idea, as in the previous activities (mentioned upper - related to the temperature measurements and graphing the temperature): "Information form the surroundings we receive via our senses, but they can deceive us." From the Big Ideas formulated by the team of Harlen (2015), we are open to developing more of them. Which we will focus on, slightly depends on the pupils. Surely, we focus on "science assumes that for every effect, there are one or more causes". It is possible that within the discussion also will arise an idea leading to the Big Ideas "Objects can affect other objects at a distance"; "Changing the movement of an object requires a net force to be acting on it"; "The total amount of energy in the Universe is always the same but energy can be transformed when things change or are made to happen"; and "Scientific explanations, theories and models are those that best fit the facts known at a particular time."

\subsection{Lesson content goals}

The goal of this activity is to develop the abilities to sense and measure the properties of objects and express them in physical quantities. We focus on the measurement of distance (length) mass, and a new dimensionless quantity. The goal is to get experience with dimensionless quantity. Also, the pupils will have experience with the quantities (compressive) force (pressing the ball), air pressure (inside the ball), volume (of the ball). A reader can say, what has been told by some teachers before they piloted this activity, that we have too many goals here. We would like to remark, this activity is focused on the mass and length measurement, and every other quantity mentioned here is presented at the level of an un-focused experience lasting a few minutes. The lesson is built on a grain size bits of curriculum, well linked together. The central context is a ball and the properties of a ball. The pupils with the teacher can discuss that a ball can be small/big (volume), hard/soft (air pressure inside the ball; force necessary to squeeze it), well / not so well bouncing (a new quantity defined in the activity).

As we mentioned earlier in this article, we are focusing this article on the exponential function development. In this particular activity, we will measure one bounce of a ball what can be seen as quite far from the exponential function. Within the grain-size knowledge development, we should say, that, later, at a higher grade, we will add more 
bounces and we will graph the maximal heights of successive bounces (via video measurement or the use of ultrasound distance sensor) and/or the times at which the ball bounces (via sound analysis). Here we examine just one rebound.

\subsection{Guiding questions for this activity}

We can guide pupils to the ideas as "Which properties of this ball we can observe?"; "Which of them can we also measure?". "At the previous lessons, we have measured the temperature of various objects. We have also measured the temperature of the ball surface. What other properties we could measure?" "You have a ball in your hand. Look at it. Feel it with your hands. Let it fall at the floor and bounce back. Observe the movement down and back. What can you measure? And how? What you would like to measure, but you don't know how?"

\subsection{Standards addressed by this activity}

Standards addressed we do not have formulated yet. This activity is a pilot activity for research on the standards for our innovated national curriculum. We would be grateful for the cooperation, with some of the readers of this contribution, on designing standards for a new physics curriculum based on The Learning Science and Knowledge in Pieces theory.

\section{Activity - "How to measure a ball?" - implemented curriculum}

The ideas planned and presented in the "intended curriculum" we projected to the workbook for pupils (Lapitkova, Tothova, Demkanin, 2019) and to the instructions for teachers. One of the authors of this contribution carried out a pilot verification on two samples of pupils - two parts of the school class. At one of the verifications, another author of this contribution was present, at the other was present a school psychologist. The sample consisted of 15 pupils in Bratislava, in October 2019.

\subsection{Setting the stage for learning}

At the beginning of the lesson, in the workbook and also in the real lesson, we focused the attention of pupils at a ball and at a broken glass window. A commix in this context is drawn at the workbook, and one of the pupils read the text of it. Also, the group of pupils presented their own measuring device - any quantity measuring device. Here was a 
difference between our intention and the real implementation - we intended to have a temperature measurement before this activity, and in the implementation, we used as a previous activity "design of own measuring device". Within the discussion, the pupils went to quantities as speed (velocity) and height - both in the context of the broken window presented in the commix.

The next part activity we can also take as an extended phase setting the stage for learning. Some balls have been shown, and the pupils generated ideas (brainstorming) on the quantities related to the balls, we could or would like to measure. The pupils discussed temperature, circumference, mass, speed (indirectly, by measuring distance and the time), volume, surface area, amount of the rubber we need to make the ball. These two groups of pupils did not mention the hardness/softness of the ball, nor the bouncing properties, what we predicted in the intended curriculum. We can assume that this was caused by the fact that the pupils at this stage did not have the balls in their hands. They had them at the table in front of them.

\subsection{Formulating investigable questions and predictions}

This activity focuses on two investigations - the mass of the ball and investigation of one bounce of a ball. Within the discussion, we have formulated some questions as "How can we find the mass of the ball? What unit is the best to use for the mass of the ball?" Looking at the digital scales, the pupils decided to use grams. The next question pupils generated was "The ball is rolling down the plate of the scales. How to keep the table tennis ball on the scales?" The solution was found soon, with a scaffold form the teacher. They used a cup, and they were able to subtract the mass of the cup from the total mass measured.

Within the bounce measurement - this was found as quite a new type of a problem. "What should we measure? 1 meter from the ground. Which part of the ball should be $1 \mathrm{~m}$ above the ground? The bottom, middle, or the highest part of the ball? How to measure the height of the ball after the bounce?"

\subsection{Planning, organising and conducting the investigation}

Within this, at a first sight simple investigation, we see, that it is not as simple as it seems. Both the mass measurement and the bounce should be well scaffolded. Pupils, with the help of the teacher, well designed general plan and also operational plan. The bounce measurement was more complex. Each of the groups of the pupils decided to measure the height of the upper point of the ball and subtract the diameter of the ball to get the height of the ball from the ground. 
Most of the groups went beyond the intended activity. They introduced a new independent variable - the initial height of the ball. After measuring bounced height for more initial heights, they tried to compare the ratio of the bounced height to initial height.

\subsection{Making meaning}

Within the classroom discussion, the pupils found that for each ball, the bounced height is always smaller than the initial height. They also found that the ratio is different for different balls. Some of the pupils concluded that this has something with energy conservation - the energy of the ball after one bounce is lower that initial energy of the ball.

\section{Conclusion}

In this contribution, we presented our view of the building knowledge by chaining a grain size knowledge. We focused on one example - one bounce measurement set to a context of measurement of properties of a ball. The pupils naturally extended the activity to measuring a bounce height for various initial heights. Building on the experience gathered within this lesson, we can later continue with graphing bounces height vs initial height, and we get a graph similar to the graph of cooling. The graph of cooling we get within a temperature measurement roughly at the same time, at the same grade. It was set in the same context the context of measuring object properties. So, the pupils will have a chance to start building a mathematical similarities chaining. Based on these two examples we plan to chain exponential character of phenomena as atmospheric pressure, epidemic, radioactive decay, beer foam decay, the amplitude of a damped oscillator, discharging the capacitor, thermistor, biological half-lives, Moore's law and probably also other. We assume that this will give enough contexts, relevant to the pupils, to develop a deep understanding of the exponential function properties. We assume the pupils will be able to use the exponential function even for settings not taught directly, as, e.g. attenuation of X-rays within a body, which we plan to use at the age of 17 .

\section{Acknowledgements}

The authors are grateful to support from the Ministry of Education of Slovak Republic, project VEGA 1/0273/19, Tutoring and Scaffolding in the Preparation of Pre-Service Physics Teachers. 


\section{References}

Demkanin, P. (2018). Didaktika fyziky pre študentov magisterského štúdia a učitel’ov v praxi, Bratislava: Univerzita Komenského v Bratislave

diSessa, A. (2014). The Construction of Casual Schemes: Learning Mechanism at the Knowledge Level, Cognitive Science, pp. 795-850.

Harlen, W. (2006). Teaching, Learning and Assessing Science 5-12, London: SAGE.

Harlen, W. (2015). Working with Big Ideas of Science Education, Trieste: Science Education Programme of IAP.

Hejny, M. (2012). Exploring the Cognitive Dimension of Teaching Mathematics trough Schemeoriented Approach to Education, In: Orbis Scholare 6(2) pp. 41-55.

Lapitková, V., Tóthová, R., Demkanin, P. (2019). Fyzika, pracovný zošit 1 pre 6. Ročník ZŠ a pre primu osemročných gymnázií, Indícia Bratislava

Sawyer, R.K. (2014). The Cambridge Handbook of The Learning Sciences, New York: Cambridge University Press

Slovák, A. (2020) Rozvijanie spôsobilostí vnímat' zákonitosti exponenciálnej funkcie vyučovaním fyziky na Š̌ a Ž̌, Unpublished bachelor thesis, Comenius University

Sfard, A., Cobb, P. (2014). Research in Mathematics Education: What Can It Teach Us about Human Learning?: In: Sawyer, R.K. The Cambridge Handbook of the Learning Sciences. 2. ed., New York: Cambridge University Press. 2014. Pp. 545 - 564. 